Snips from the Journals

\title{
Tragedies waiting to happen? Unravelling potential psychiatric repercussions during the COVID-19 pandemic
}

\author{
SR Perera, A Wickramasinghe
}

To date, the 2019-coronavirus disease (COVID-19), has claimed more than 1.4 million deaths globally, and is undoubtedly the greatest public health catastrophe of our times (1). The adverse effects of this pandemic on the mental health are already being well documented, with a recent study demonstrating that one in five COVID19 patients are diagnosed with a psychiatric disorder such as anxiety or depression within three months of testing positive for the virus $(2,3)$. This situation is further aggravated by disruption to the provision of services to these people in the community (4).

\section{Impact of COVID-19 pandemic on mental health in the general population: A systematic review (5)}

The rapidly evolving, unexpected, ongoing pandemic is changing all our lives in many different ways. The authors of this article conducted a systematic review to examine the prevalence of, and factors associated with, symptoms of depression, anxiety, posttraumatic stress disorder (PTSD) and other forms of psychological distress in the general population during the COVID-19 pandemic (5). They conducted a systematic search on several recognized electronic databases including PubMed, Medline, Embase, Scopus and the Web of Science, and included all relevant articles published upto $17^{\text {th }}$ May 2020, in either English or Chinese. The final review included 19 published studies.

The authors report that the studies revealed a comparatively high rate of symptoms of anxiety (6.3\%-50.9\%), depression (14.6\%-48.3\%), PTSD (7\%-53.8\%) and psychological distress (34.4\%-38\%). Features of depression were more common among females compared to males, younger people ( $<40$ years) and students. Similarly, anxiety was also more common among younger people, and was associated with other factors such as female gender, lower level of education, poor self-rated levels of health, loneliness, quarantine status, worry about infection, and prior mental health or physical health problems. Furthermore, frequent exposure to news/ information about COVID-19 and social media was also associated with increased symptoms of anxiety.
The review also identified a few studies that had examined for factors that protected against psychological illness. These protective factors included: Timely dissemination of updated and accurate information by the authorities, and actively carrying out measures to lower risk of infection on the part of the individual (such as mask wearing and hand-washing). Individuals with positive coping styles also appeared to have fewer symptoms of anxiety and stress.

Limitations of this review included the heterogeneity of the studies included, and also the fact that almost all the studies included in the review were conducted in developed countries.

The authors conclude by noting that a high prevalence of adverse psychiatric symptoms were noted in most studies, and they suggest prioritization of prevention of common mental disorders such depression, anxiety, as well as suicide, during the COVID-19 pandemic. They recommend that policymakers prioritize vulnerable groups, and ensure timely and accurate dissemination of information regarding the pandemic situation, in order to minimize rumors and false information, which perpetuates anxiety. The importance of provision of accessible mental health care was also noted, particularly for people from rural areas who face travel restrictions, as well as for those in quarantine.

\section{Neurological and neuropsychiatric compli- cations of COVID-19 in 153: a UK-wide surveillance study (6)}

Given the possible neuropsychiatric complications of COVID-19, this study aimed to investigate the complications of COVID-19 that affected the brain, as identified in the United Kingdom. This was a cross sectional study, describing a 'snapshot' of the neuropsychiatric sequelae associated with COVID-19, reported across UK, during the month of April 2020. Data was collected via an online network of secure rapidresponse case report notification across several major UK neuroscience bodies, including the Royal College of Psychiatrists and the Association of British Neurologists. The authors describe data on 125 COVID-19 patients with neuropsychiatric manifestations, reported during the study period. 
A majority of reported patients (62\%) had experienced a cerebrovascular accident (most often an ischaemic stroke), and these were mainly older patients. However 39 (31\%) had presented with altered mental status which included nine (23\%) of patients with unspecified encephalopathy, and seven (18\%) with encephalitis. The remaining 23 patients presented with altered mental status, of whom, ten were diagnosed to be suffering from a new-onset psychosis, six had a neurocognitive syndrome and four had an affective disorder.

The authors note that altered mental status was the second most common diagnoses, comprising encephalopathy, encephalitis or a primary psychiatric diagnosis, and this was more common among younger patients.

The limitations of this study include the narrow time frame, and the consideration of inpatients only, representing those with more severe COVID-19 symptoms. Furthermore, given the cross-sectional nature of the study, it needs to be emphasized that a causal nature of association between COVID-19 and the reported neuropsychiatric presentations cannot be established based on the findings of this study. Increased awareness about this important area, and further research to test possible causal relationships between COVID-19 and neurological and psychiatric symptoms, is indicated.

Children and adolescents are probably more likely to experience high rates of depression and anxiety in the aftermath of COVID-19 (7)

Governments the world over have taken stringent containment measures which include closure of schools, social distancing and home quarantine in response to the COVID-19 spread (7). Loades et al. and others hypothesize that these measures may increase mental health problems in children and adolescents $(7,8)$. Loneliness is reported to be associated with future mental health problems up to 9 years later, and has a strong association with the development of depression (7). Furthermore, loneliness was found to be associated with elevated symptoms of depression in girls and elevated social anxiety in boys, and the length of loneliness appears to be a predictor of future mental health problems (7). The authors note that this carries a special significance within the COVID-19 context, as policy makers in different countries debate the length of time that schools should remain closed and discuss the implementation of social distancing within schools (7). The authors also highlight the importance of offering preventive support and early intervention where possible, and the need to be prepared for an increase in mental health problems in children and adolescents (7).
Addictive behaviors and COVID-19 form dangerous partners (9)

This review highlights the bi-directional relationship between COVID-19 and addiction and analyzes how these two "menaces" interact with each other to impose a greater public health threat.

This review notes that people with substance use disorders (SUDs) are at heightened risk for pulmonary infections due to pre-existing cardio-pulmonary morbidities, compromised anatomy and immunological functions, altered health-seeking behavior and inadequate access and failure of rehabilitation strategies (9). Smoking has been found to be an adverse prognostic indicator of COVID-19, apart from traditional risks imposed by smoking on cardio-pulmonary health (10).

A similar health-risk might be imposed by alcohol consumption, although there is lack of systematic data regarding alcohol consumption and COVID-19 risk (9). Those with alcohol use have a range of medical problems including vitamin deficiency, heightened risk of aspiration pneumonia and associated liver diseases, which may act synergistically to cause or worsen health outcomes (10).

The authors also highlight the risks regarding opioid misuse - those who take opioids for various medical conditions are at increased risk of dangerous overdosage, which can cause respiratory depression and hypoxemia, leading to cardiopulmonary and neurological complications which worsen the outcome of COVID-19; while those dependent on opiates are facing severe challenges at this time due to greater marginalization, difficulty in accessing specific healthcare, imposed restriction on drugs, closure of de-addiction centers, increased risk of life- threatening withdrawal and usage of illicit opiates (11).

Methamphetamines cause lung injury, pulmonary hypertension, and cardiomyopathy. Experts have advised vigilance regarding the possibility of increased risk of worse COVID-19 outcomes among abusers of methamphetamine (12). Those in distress may take refuge in addictive substances to allay their negative feelings, which could potentially trigger the development of SUDs in high-risk groups and a spike in incidence of SUDs among general population as well (13).

Apart from the surge in SUDs, the review draws attention to an associated "infodemic" and internet addiction that have surfaced in this unprecedented time (12). Evidence indicates that usage of the internet, particularly websites related to pornography and video gaming, has markedly increased in this period of lockdown (14). 
Lockdown, domestic violence, mental illness and COVID-19: Reaching out to those in need (15)

Domestic violence is a largely hidden epidemic - and never more so than in this current state of mandatory seclusion (16). Addressing domestic violence is a global public health priority, given the prevalence of domestic violence and the associated psychological and physical morbidity and mortality (17). Increased concerns about domestic violence have been expressed in many countries with the travel restrictions imposed to prevent the spread of COVID-19 (18). Other reasons include social isolation, exposure to economic and psychological stressors, increase in negative coping mechanisms (such as alcohol misuse) and an inability to access usual support or escape abusive households, owing to quarantine or travel restrictions (19).

While previous research has suggested an association between mental illness and the perpetration of domestic violence, it should be reiterated that substance use disorders carry the highest absolute and relative risks of domestic violence (18). Therefore treatment for the underlying substance use disorder, alongside any coexisting mental illness should be prioritized in order to reduce the risk (19). Furthermore, people with severe mental illness are at increased risk of becoming victims of domestic violence, although the reported numbers may not reflect the actual numbers because those with mental illness are generally reluctant to disclose abuse (20). Well recognized reasons for non-disclosure include the fear of further violence, fear of social services involvement and worry that they would not be believed (21).

Despite a probable increase in psychological distress, victimisation and mental illness, it is likely that many people may not seek help owing to fears that services are overwhelmed and that attending face-to-face appointments might put them at risk (22). It is therefore, particularly important that mental illness is recognized at primary care level and that onward referrals are made to specialist services. There are concerning reports of staff re-deployment from psychiatric services to other health services in response to the pandemic (23). This may disrupt continuity of care, leading to a lack of consistent and assertive follow-up, especially among those who depend on psychiatric services for safety and advocacy.

\section{Conflicts of interest}

None declared.
SR Perera, Department of Psychiatry, Faculty of Medicine, University of Peradeniya, Sri Lanka

A Wickaremasinghe, Department of Psychiatry, Faculty of Medicine and Allied Sciences, Rajarata University of Sri Lanka.

Corresponding author: SR Perera

Email: sayuperera@yahoo.com

http://orcid.org/0000-0001-6824-8991

\section{References}

1. World Health Organization (WHO). Coronavirus disease (COVID-2019) Situation report [Internet]. WHO 2020 [cited 2020 Apr 20]. Available from: https://www.who.int/ emergencies/diseases/novel-coronavirus-2019/situationreports

2. Rajkumar RP. COVID-19 and mental health: A review of the existing literature. Asian J Psychiatr. 2020; 10: 10206.

3. The Lancet Infectious Diseases. The intersection of COVID-19 and mental health. Lancet Infect Dis. 2020; 20(11): 1217

4. Klomek AB. Suicide prevention during the COVID-19 outbreak. Lancet Psychiatry. 2020; 7(5): 390.

5. Xiong J, Lipsitz O, Nasri F, Lui LMW, Gill H, Phan L, Chen-Li D, Iacobucci M, Ho R, Majeed A, McIntyre RS. Impact of COVID-19 pandemic on mental health in the general population: A systematic review. J Affect Disord 2020; 277: 55-64.

6. Varatharaj A, Thomas N, Ellul MA, Davies NWS, Pollak TA, Tenorio EL, et al. Neurological and neurophsychiatric complications of COVID-19 in 153: a UK-wide surveillance study. Lancet Psychiatry 2020; 7: 875-82.

7. Loades ME, Chatburn E, Higson-Sweeney N, Reynolds S, Shafran R, Brigden A, Linney C, McManus MN, Borwick C, Crawley E. Rapid Systematic Review: The Impact of Social Isolation and Loneliness on the Mental Health of Children and Adolescents in the Context of COVID-19. Jour Am Acad Child \& Adol Psych 2020.

8. Brooks SK, Webster RK, Smith LE, et al. The psychological impact of quarantine and how to reduce it: rapid review of the evidence. Lancet. 2020; 395(10227): 912.

9. Dubey MJ, Ghosh R, Chatterjee S, Biswas P, Chatterjee S, Dubey S. COVID-19 and addiction. Diabetes \& Metabolic Syndrome Clin Res \& Rev 2020.

10. Vardavas CI, Nikitara K. COVID-19 and smoking: A systematic review of the evidence. Tob Induc Dis. 2020; 18: 20.

11. Testino G. Are Patients With Alcohol Use Disorders at Increased Risk for Covid-19 Infection? Alcohol Alcohol. 2020; 55(4): 344-6. 
12. Becker WC, Fiellin DA. When Epidemics Collide: Coronavirus Disease 2019 (COVID-19) and the Opioid Crisis. Ann Intern Med. 2020; 173(1): 59.

13. Volkow ND. Collision of the COVID-19 and Addiction Epidemics. Ann Intern Med. 2020; 173(1): 61-2.

14. Király O, Potenza MN, Stein DJ, et al. Preventing problematic internet use during the COVID-19 pandemic: Consensus guidance. Compr Psychiatry. 2020; 100: 152180.

15. Gulati G, Kelly BD. Domestic violence against women and the COVID-19 pandemic: What is the role of psychiatry?. Int Jour Law \& Psych. 2020: 101594.

16. Kofman YB, Garfin DR. Home is not always a haven: The domestic violence crisis amid the COVID-19 pandemic. Psychol Trauma. 2020; 12(S1): S199-S201.

17. Bradbury-Jones $C$, Isham L. The pandemic paradox: The consequences of COVID-19 on domestic violence. J Clin Nurs. 2020; 29(13-14): 2047-2049
18. Vora M, Malathesh BC, Das S, Chatterjee SS. COVID-19 and domestic violence against women. Asian J Psychiatr. 2020; 53: 102227.

19. Rund BR. A review of factors associated with severe violence in schizophrenia. Nord J Psychiatry. 2018; 72(8): 561-71.

20. Khalifeh H, Oram S, Trevillion K, Johnson S, Howard LM. Recent intimate partner violence among people with chronic mental illness: findings from a national crosssectional survey. Br J Psychiatry. 2015; 207(3): 207-12.

21. Rose D, Trevillion K, Woodall A, Morgan C, Feder G, Howard L. Barriers and facilitators of disclosures of domestic violence by mental health service users: qualitative study. Br J Psychiatry. 2011; 198(3): 189-94.

22. Kelly BD. Coronavirus disease: challenges for psychiatry. Br J Psychiatry. 2020; 217(1): 352-3.

23. Cullen W, Gulati G, Kelly BD. Mental health in the COVID-19 pandemic. QJM. 2020; 113(5): 311-12. 